\title{
Erratum to: Geant4-based electromagnetic background model for the CRESST dark matter experiment
}

\author{
A. H. Abdelhameed ${ }^{1}$, G. Angloher ${ }^{1}$, P. Bauer ${ }^{1}$, A. Bento ${ }^{1,9}$, E. Bertoldo ${ }^{1}$, R. Breier ${ }^{2}$, C. Bucci ${ }^{3}$, L. Canonica ${ }^{1}$, \\ A. D’Addabbo ${ }^{3,10}$, S. Di Lorenzo ${ }^{3,10}$, A. Erb $^{4,11}$, F. v. Feilitzsch ${ }^{4}$, N. Ferreiro Iachellini ${ }^{1}$, S. Fichtinger ${ }^{5}$, A. Fuss ${ }^{5,6}$, \\ P. Gorla ${ }^{3}$, D. Hauff ${ }^{1}$, M. Ješkovský ${ }^{2}$, J. Jochum ${ }^{7}$, J. Kaizer ${ }^{2}$, A. Kinast ${ }^{4}$, H. Kluck ${ }^{5,6, a}{ }^{(D,}$, H. Kraus ${ }^{8}$, \\ A. Langenkämper ${ }^{4}$, M. Mancuso ${ }^{1}$, V. Mokina ${ }^{5, b}{ }_{(D)}$, E. Mondragón ${ }^{4}$, M. Olmi ${ }^{3,10}$, T. Ortmann ${ }^{4}$, C. Pagliarone $^{3,12}$,

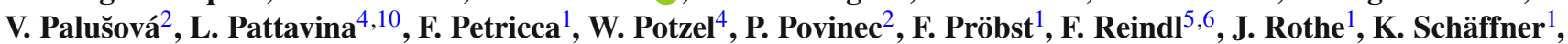 \\ J. Schieck ${ }^{5,6}$, V. Schipperges ${ }^{7}$, D. Schmiedmayer ${ }^{5,6}$, S. Schönert ${ }^{4}$, C. Schwertner ${ }^{5,6}$, M. Stahlberg ${ }^{5,6}$, L. Stodolsky $^{1}$, \\ C. Strandhagen ${ }^{7}$, R. Strauss ${ }^{4}$, C. Türkoğlu ${ }^{5,6,14, c}$ (D), I. Usherov ${ }^{7}$, M. Willers ${ }^{4}$, V. Zema ${ }^{3,10,13}$, J. Zeman ${ }^{2}$ \\ ${ }^{1}$ Max-Planck-Institut für Physik, 80805 Munich, Germany \\ 2 Faculty of Mathematics, Physics and Informatics, Comenius University, 84248 Bratislava, Slovakia \\ ${ }^{3}$ INFN, Laboratori Nazionali del Gran Sasso, 67100 Assergi, Italy \\ ${ }^{4}$ Physik-Department and Excellence Cluster Universe, Technische Universität München, 85747 Garching, Germany \\ ${ }^{5}$ Institut für Hochenergiephysik der Österreichischen Akademie der Wissenschaften, 1050 Wien, Austria \\ ${ }^{6}$ Atominstitut, Technische Universität Wien, 1020 Wien, Austria \\ ${ }^{7}$ Eberhard-Karls-Universität Tübingen, 72076 Tübingen, Germany \\ ${ }^{8}$ Department of Physics, University of Oxford, Oxford OX1 3RH, UK \\ ${ }^{9}$ Departamento de Fisica, Universidade de Coimbra, 3004516 Coimbra, Portugal \\ ${ }^{10}$ GSSI-Gran Sasso Science Institute, 67100 L'Aquila, Italy \\ ${ }^{11}$ Walther-Meißner-Institut für Tieftemperaturforschung, 85748 Garching, Germany \\ 12 Dipartimento di Ingegneria Civile e Meccanica, Universitá degli Studi di Cassino e del Lazio Meridionale, 03043 Cassino, Italy \\ 13 Department of Physics, Chalmers University of Technology, SE-412 96 Göteborg, Sweden \\ ${ }^{14}$ Present address: School of Mathematical and Physical Sciences, University of Sussex, Brighton BN1 9QH, UK
}

Published online: 4 December 2019

(C) The Author(s) 2019

Erratum to: Eur. Phys. J. C (2019) 79:881 https://doi.org/10.1140/epjc/s10052-019-7385-0

The original version of this article unfortunately contains mistakes in the PDF on pages 10, 12, 15, 16 and 17. Due to typesetting mistakes in Sect. 4.2, first paragraph, and in the table headers of Tables 4, 6, 7, 8, 9, 10 and 11 it reads "mBq" instead of " $\mu \mathrm{Bq}$ ".
Open Access This article is distributed under the terms of the Creative Commons Attribution 4.0 International License (http://creativecomm ons.org/licenses/by/4.0/), which permits unrestricted use, distribution, and reproduction in any medium, provided you give appropriate credit to the original author(s) and the source, provide a link to the Creative Commons license, and indicate if changes were made. Funded by $\mathrm{SCOAP}^{3}$.

The original article can be found online at https://doi.org/10.1140/ epjc/s10052-019-7385-0.

\footnotetext{
a e-mail: holger.kluck@oeaw.ac.at

b e-mail: valentyna.mokina@oeaw.ac.at

c e-mail: c.turkoglu@sussex.ac.uk
} 